\title{
Performance comparison of neural network training methods based on wavelet packet transform for classification of five mental tasks
}

\author{
Vijay Khare $^{1}$, Jayashree Santhosh ${ }^{2}$, Sneh Anand ${ }^{3}$, Manvir Bhatia ${ }^{4}$ \\ ${ }^{1}$ Jaypee Institute of Information Technology, Department of Electronics and communication Engineering, New Delhi, India; \\ ${ }^{2}$ Indian Institute of Technology, Computer Services Centre, New Delhi, India; \\ ${ }^{3}$ Indian Institutes of Technology, Centre for Biomedical Engineering, New Delhi, India; \\ ${ }^{4}$ Department of Sleep Medicine, New Delhi, India. \\ Email: vijay.khare@jiit.ac.in; jayashree@cc.iitd.ac.in; sneh@iitd.ernet.in; manvirbhatia1@yahoo.com
}

Received 11 February 2010; revised 15March 2010; accepted 25 March 2010.

\begin{abstract}
In this study, performances comparison to discriminate five mental states of five artificial neural network (ANN) training methods were investigated. Wavelet Packet Transform (WPT) was used for feature extraction of the relevant frequency bands from raw electroencephalogram (EEG) signals. The five ANN training methods used were (a) Gradient Descent Back Propagation (b) Levenberg-Marquardt (c) Resilient Back Propagation (d) Conjugate Learning Gradient Back Propagation and (e) Gradient Descent Back Propagation with movementum.
\end{abstract}

Keywords: Electroencephalogram (EEG); Wavelet Packet Transform (WPT); Artificial Neural Network (ANN)

\section{INTRODUCTION}

Brain computer interface use a non muscular communication channel for conveying message and command to the external words in the absence of biological channels [1-3]. Neuromuscular disorders like Amyotrophic lateral sclerosis can temporarily or permanently impair spoken and physical communication. Those most severely affected may lose all voluntary muscle control and may be completely locked in to their bodies, unable to communicate in any way. Using cognitive abilities is sometimes the only the way to restore communication and motor function [4-7]. Through training, subjects can learn to control their brain activity in predefined fashion that is classified by pattern recognition algorithms. Accuracy of classification is affected by the quality of EEG signals and the processing algorithms. The processing algorithms include preprocessing, feature extraction and classification. Previous studies investigated the effect of different feature extraction algorithms with along and the different mental tasks on classification accuracy was investigated $[8,9]$.

In this study, wavelet packet transform (WPT) method was used to capture the information of mental tasks from eight channel EEG signals of nine subjects. The coefficients of wavelet packet transform (WPT) were used as the best fitting input vector for ANN. Five various artificial neural networks (ANN) training methods were used to compare the performance in discrimination of five mental tasks.

\section{METHODOLOGY}

\subsection{Subjects}

Nine right-handed healthy male subjects of age (mean $23 y r$ ) having no sign of any motor- neuron diseases were selected for the study. A pro-forma was filled in with detail of their age \& education level. The participants were student volunteers for their availability and interest in the study. EEG data was collected after taking written consent for participation. Full explanation of the experiment was provided to each of the participants.

\subsection{EEG Data Acquisition}

EEG Data used in this study was recorded on a Grass Telefactor EEG Twin3 Machine available at Deptt. of Neurology, Sir Ganga Ram Hospital, New Delhi. EEG recording was done for five mental tasks for five days, from nine selected subjects. Data was recorded for 10 sec during each task and each task was repeated five times per session per day. Bipolar and Referential EEG was recorded using eight standard positions $\mathrm{C} 3, \mathrm{C} 4, \mathrm{P} 3$, $\mathrm{P} 4, \mathrm{O} 1 \mathrm{O} 2$, and $\mathrm{F} 3, \mathrm{~F} 4$ by placing gold electrodes on scalp, as per the international 10-20 standard system of electrode placement as shown in Figure 1. The settings 


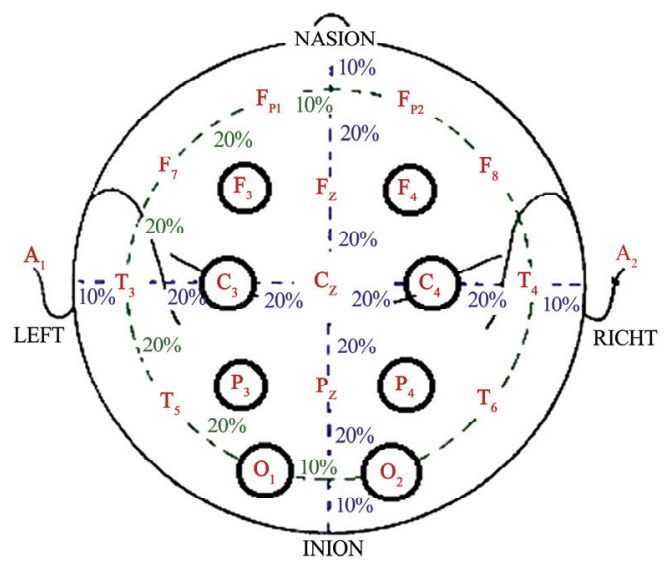

Figure 1. Montage for present study.

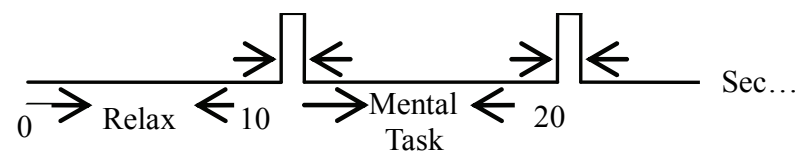

Figure 2. Timing of the Protocol.

used for data collection were: low pass filter $1 \mathrm{~Hz}$, high pass filter $35 \mathrm{~Hz}$, sensitivity 150 micro volts $/ \mathrm{mm}$ and sampling frequency fixed at $400 \mathrm{~Hz}$. The reference electrodes were placed on ear lobes and ground electrode on the forehead. EOG (Electooculargram) being a noise artifact, was derived from two electrodes placed on outer canthus of left and right eye in order to detect and eliminate eye movement artifacts.

\subsection{Experiment Paradigm}

An experiment paradigm was designed for the study and the protocol was explained to each participant before the experiment. In this, the subject was asked to comfortably lie down in a relaxed position with eyes closed. After assuring the normal relaxed state by checking the status of alpha waves, the EEG was recorded for $50 \mathrm{sec}$, collecting five session of $10 \mathrm{sec}$ epoch for the relaxed state. This was used as the baseline reference for further analysis of mental task. The subject was asked to perform a mental task on presentation of an audio cue. Five session of $10 \mathrm{sec}$ epoch for each mental task were recorded as shown in Figure 2. The whole experimental lasted for about one hour including electrodes placement.

Data collected from nine subjects performing five mental tasks were analyzed. The following mental tasks were used:

Relaxed: The subject was asked to relax with their eyes closed. No mental or physical task to be performed at this stage.

Arithmetic Task: The subject was asked to perform simple arithmetic (SA) and complex arithmetic (CA). An example of a trivial calculation is to multiply 2 by 3 and nontrivial task is to multiply 49 by 78 . The subject was instructed not to vocalize or make movements while solving the problem. EEG signal were recorded corresponding.

Geometric Figure Rotation (R): The subject was given 30 seconds to see complex three dimensional objects, after which the object was removed. The subject was instructed to visualize the object being rotated about an axis. The EEG signals were recorded during this period.

Movement Imagery (M): The subject was asked to plan movement of the right hand and corresponding EEG signals were recorded during this period.

\subsection{Feature Extraction}

The frequency spectrum of the signal was first analyzed through Fast Fourier Transform (FFT) method [10]. The FFT plot of signals from the all electrode pairs were observed and maximum average change in EEG amplitude was noted as shown in Figures 3-7.

For relaxed, the peaks of power spectrum almost coincide for central area in the alpha frequency range (8-13 $\mathrm{Hz})[11]$. EEG recorded with relaxed state is considered to be the base line for the subsequent analysis. $\mathrm{Mu}$ rhythms are generated over sensorimotor cortex during planning a movement. For movement imagery (M) of right hand, maximum up to $50 \%$ band power attenuation was observed in contralateral (C3 w.r.t C4) hemisphere in the alpha frequency range $(8-13 \mathrm{~Hz})[11,12]$. For geometrical figure rotation(R), the peak of the power spectrum was increased in right hemisphere rather than left in the occipital area for the alpha frequency range (8-13 $\mathrm{Hz}$ ) [13]. For simple arithmetic(SA), the peak of the power spectrum was increased in left hemisphere rather than right hemisphere in the frontal area for the alpha frequency range $(8-13 \mathrm{~Hz})$ [14].For complex arithmetic (CA), the peak of the power spectrum was increased in left hemisphere rather than right hemisphere in the parietal area for the alpha frequency range $(8-13 \mathrm{~Hz})$.

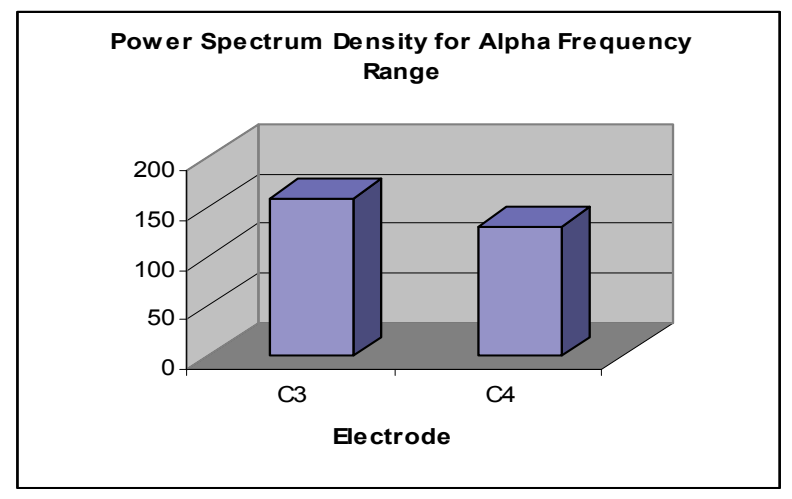

Figure 3. Power Spectra for Relax state at C3 and C4 channel. 


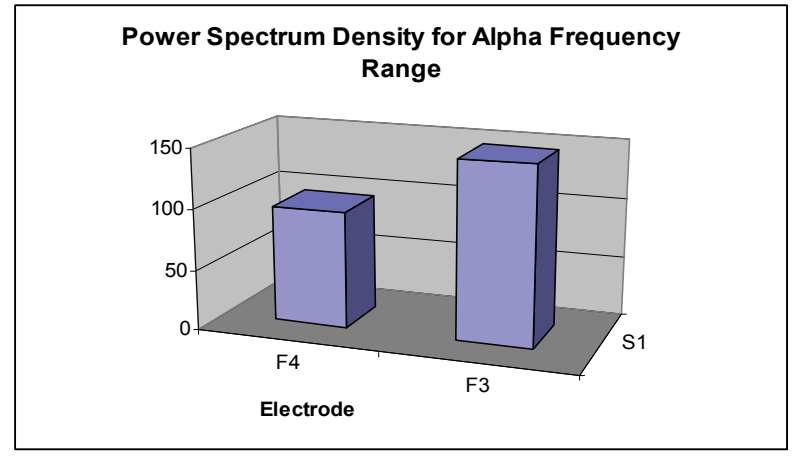

Figure 4. Power Spectra for simple arithmetic at F3 and F4 channel.

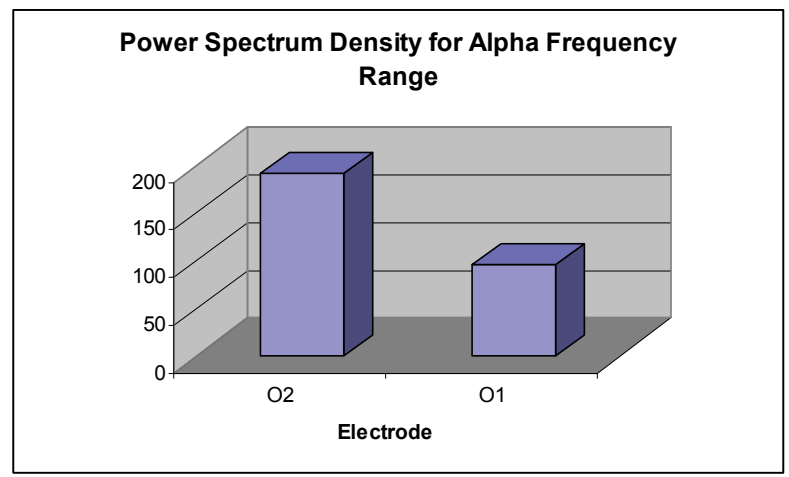

Figure 5.Power Spectra for rotation at $\mathrm{O} 1$ and $\mathrm{O} 2$ channel.

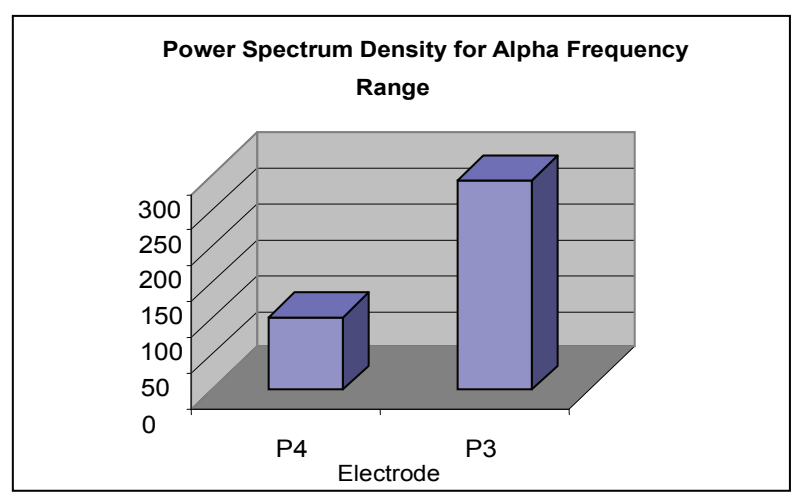

Figure 6. Power Spectra for complex Arithmetic P3 and P4 channel.

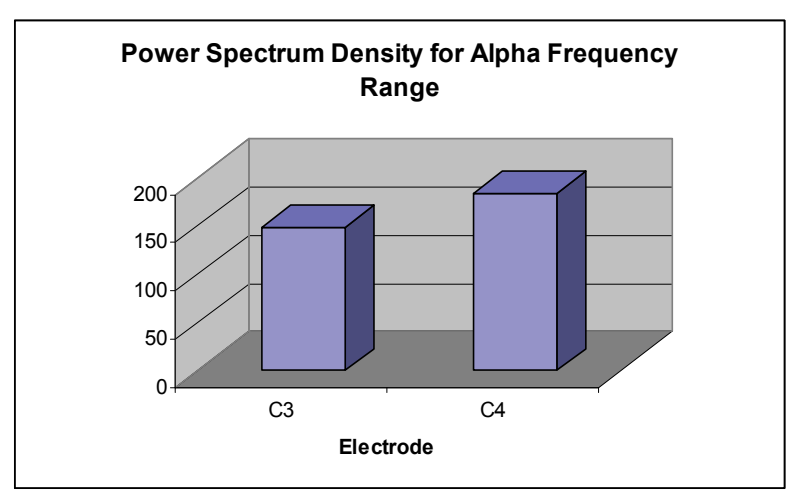

Figure 7. Power Spectra for right movement at $\mathrm{C} 3$ and $\mathrm{C} 4$ channel.

\subsection{Wavelet Packet Transform}

By applying Wavelet packet analysis on the original signal wavelet coefficients in the 8-13 HZ frequency band at the $5^{\text {th }}$ level node $(5,3)$ were obtained. The signal was reconstructed at node $(5,3)$. These coefficients are scaled and WPT coefficients are used as the best fitting input vector for ANN. Wavelet transform we were able to reduce 1 second of EEG data to 21 coefficients for each mental tasks $[15,16]$.

\subsection{Classification}

The main advantage of choosing artificial neural network for classification was due to fact that ANN's could be used to solve problems, where description for the data is not computable. ANN could be trained using data to discriminate the feature. The five different training methods used for Classification in the present study were Gradient Descent method Resilient Back propagation, Levenberg-Marquardt, Conjugate Gradient Descent and Gradient Descent back propagation with movementum.

For classification a two layer neural networks was used for the instance a topology of $\{10,1\}$ indicate a 21 input, 10 neurons in hidden layer and one output layer. The neural network was designed to accept a 21 element input vector and give a single output. One second segments of EEG were classified. The training set was composed of $60 \%$ EEG trial per mental tasks and test set was composed $40 \%$ EEG trial per mental task. The output was designed to give 0 for baseline and 1 for task. The neural network was trained for a fixed number of epochs and the training is done using a five learning techniques [17]. Parameter used for five training methods of neural network for classification of five mental tasks as shown in the Table 1.

\subsection{Evaluations}

Performance $\left(\mathrm{R}_{\mathrm{C}}\right)$ is defined as ratio between correctly classified patterns in the test set to the total number of patterns in the test set in percentage.

$$
\mathrm{Rc}=\frac{\text { Number of correctly classified test patterns }}{\text { Total number of patterns in the test set }}
$$

With the help of above formula we calculate the performance of each method for each task [18].

\section{RESULTS}

For the classification of five mental tasks neural network training methods were used. Nine male right-handed subjects participated in the experiments. The subjects asked to perform five mental tasks namely relaxed, arithmetic task (simple and complex multiplication), geometrical figure rotation, and movement imagery. Table 2 showed the comparison of the performance of five neu- 
Table1. Parameter used for different back propagation algorithms with topology $\{10,1\}$.

\begin{tabular}{|c|c|}
\hline \multicolumn{2}{|c|}{ Gradient Descent with Momentum (GDM) } \\
\hline Topology $\{10,1\}$ & $\alpha=0.01 . \mathrm{Mu}=0.01$ \\
\hline \multicolumn{2}{|l|}{$\mathrm{MSE}=1 \mathrm{e}-5$} \\
\hline \multicolumn{2}{|l|}{ Epoach $=5000$} \\
\hline \multicolumn{2}{|c|}{ Gradient Descent method (GD) } \\
\hline Topology $\{10,1\}$ & $\alpha=0.01$ \\
\hline \multicolumn{2}{|l|}{ MSE = 1exp-(5) } \\
\hline \multicolumn{2}{|l|}{ Epoach $=5000$} \\
\hline \multicolumn{2}{|c|}{ Resilient Back propagation (RBP) } \\
\hline Topology $\{10,1\}$ & $\alpha=0.01$ \\
\hline \multicolumn{2}{|l|}{ MSE = 1exp-(5) } \\
\hline Epoach $=5000$ & $\beta=0.75$ and $\beta_{1}=1.05$ \\
\hline \multicolumn{2}{|c|}{ Conjugate Gradient descent (CG) } \\
\hline Topology $\{10,1\}$ & $\alpha=0.01$ \\
\hline \multicolumn{2}{|l|}{ MSE = 1exp-(5) } \\
\hline \multicolumn{2}{|l|}{ Epoach $=5000$} \\
\hline \multicolumn{2}{|c|}{ Levenberg-Marquardt (LM) } \\
\hline Topology $\{10,1\}$ & $\mathrm{Mu}=0.01$ \\
\hline \multicolumn{2}{|l|}{ MSE = 1exp-(5) } \\
\hline Epoach $=5000$ & Mu_dec $=0.1$ and Mu_inc $=10$ \\
\hline
\end{tabular}

ral network (NN) training methods in classifying of five mental tasks. From this table we can say that Resilient Back Propagation method was most suitable for the classification of all five mental tasks. Because this method gave highest performance (95\%) all five mental tasks. Percentage average accuracy shown in the Figures 812.

\section{CONCLUSIONS}

In this study, nine healthy male subjects were selected to investigate MLP classifier with various training methods to discriminate five mental tasks (relaxed state, movement imagery of right hand, geometrical figure rotation, arithmetic simple task, and arithmetic complex task) effectively. For relaxed, the peaks of power spec-

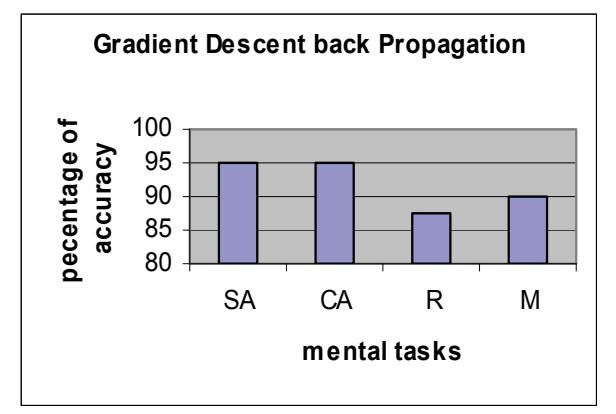

Figure 8. Classification accuracy using GDA BP training methods. trum almost coincide in central area at a particular based frequency. For simple arithmetic, it was observed that the amplitude of the power spectrum for alpha frequency range $(8-13 \mathrm{~Hz})$ increased left hemisphere rather than right hemisphere in frontal region. For complex arithematic, it was observed that the amplitude of the power spectrum for alpha frequency range $(8-13 \mathrm{~Hz})$ increased

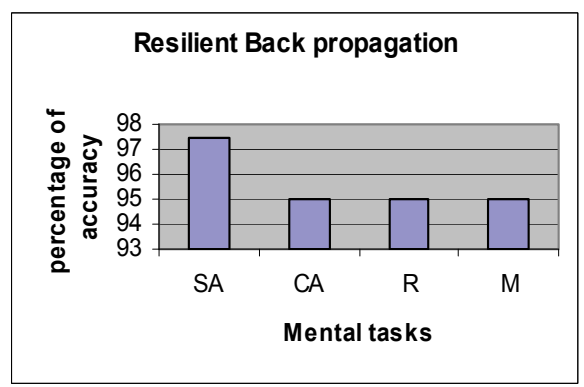

Figure 9. Classification accuracy RBP training methods.

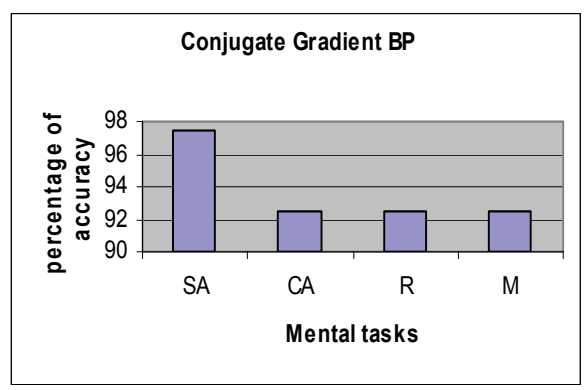

Figure 10. Classification accuracy CGBP training methods.

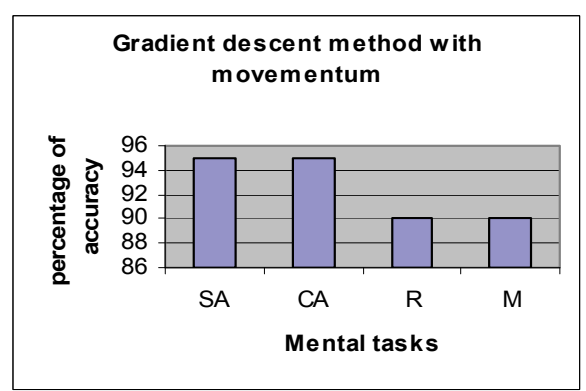

Figure 11. Classification accuracy GDM training methods.

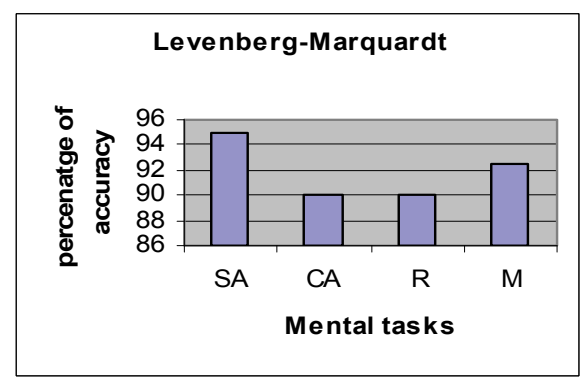

Figure 12. Classification accuracy LM training methods. 
Table 2. Comparisons of Different NN training Methods.

\begin{tabular}{|c|c|c|c|c|c|c|c|c|}
\hline \multirow{2}{*}{$\begin{array}{c}\text { Tasks } \\
\text { Techniques }\end{array}$} & \multicolumn{2}{|c|}{$\begin{array}{c}\text { Baseline and } \\
\text { Arithmetic simple }\end{array}$} & \multicolumn{2}{|c|}{$\begin{array}{l}\text { Baseline and Arithmetic } \\
\text { complex }\end{array}$} & \multicolumn{2}{|c|}{ Baseline and Rotation } & \multicolumn{2}{|c|}{ Baseline and movement } \\
\hline & $\begin{array}{c}\text { Correct } \\
\text { classification }\end{array}$ & $\begin{array}{c}\text { Wrong } \\
\text { classifica- } \\
\text { tion }\end{array}$ & $\begin{array}{l}\text { Correct } \\
\text { classifica- } \\
\text { tion }\end{array}$ & $\begin{array}{l}\text { Wrong } \\
\text { classifica- } \\
\text { tion }\end{array}$ & $\begin{array}{l}\text { Correct } \\
\text { classifica- } \\
\text { tion }\end{array}$ & $\begin{array}{l}\text { Wrong } \\
\text { classifi- } \\
\text { cation }\end{array}$ & $\begin{array}{l}\text { Correct } \\
\text { classifica- } \\
\text { tion }\end{array}$ & $\begin{array}{c}\text { Wrong } \\
\text { classifica- } \\
\text { tion }\end{array}$ \\
\hline $\begin{array}{c}\text { Gradient Descent Back } \\
\text { Propagation }\end{array}$ & $95 \%$ & $5 \%$ & $95 \%$ & $5 \%$ & $87.5 \%$ & $12.5 \%$ & $90 \%$ & $10 \%$ \\
\hline Resilient Back Propagation & $97.5 \%$ & $2.5 \%$ & $95 \%$ & $5 \%$ & $95 \%$ & $5 \%$ & $95 \%$ & $5 \%$ \\
\hline Conjugated Gradient BP & $97.5 \%$ & $2.5 \%$ & $92.5 \%$ & $7.5 \%$ & $92.5 \%$ & $7.5 \%$ & $92.5 \%$ & $7.5 \%$ \\
\hline GD BP with Momentum & $95 \%$ & $5 \%$ & $95 \%$ & $5 \%$ & $90 \%$ & $10 \%$ & $90 \%$ & $10 \%$ \\
\hline Levenberg-Marquardt & $95 \%$ & $5 \%$ & $90 \%$ & $10 \%$ & $90 \%$ & $10 \%$ & $92.5 \%$ & $7.5 \%$ \\
\hline
\end{tabular}

left hemisphere rather than right hemisphere in parietal region. For geometrical figure rotation, the peak of the power spectrum in the alpha frequency range $(8-13 \mathrm{~Hz})$ increased right occipital area. For movement imagery, the peak of the power spectrum in the alpha frequency range $(8-13 \mathrm{~Hz})$ had an attenuation central area. The result showed the performance of neural network with various training method, for classifying of mental tasks w.r.t baseline. Resilient backpropagation training method has best performance among all the training methods for classification of mental tasks w.r.t baseline.

The authors would like to extend the work with severely disabled people and to customize the device as per individual response and requirements. This kind of system can also be used in a variety of applications likeEnvironment control units (ECU'S), helping disable people to directly interact with hand held devices such as cell phones and PDAs.

\section{ACKNOWLEDGEMENTS}

The authors would like to acknowledge their gratitude to the Centre of Biomedical Engineering of IIT New Delhi and Electronics and communication department of JIIT Noida. The authors also thank the scientific and technical staff of EEG Laboratory of Sir Ganga Ram hospital, New Delhi for the help in carrying out the experiment.

\section{REFERENCES}

[1] Wolpaw, J.R., Birbaumer, N., Mc Farland, D.J., Plurtscheller, G. and Vaughan, T.M. (2002) Brain computer interfaces for communication and control. Clinical Neurophysiology, 113(6), 767-791.

[2] Pfurtschelle, G., Flotzinger, D. and Kalcher, J. (1993) Brain computer interface-A new communication device for handicapped people. Journal of Microcomputer Applications, 16, 293-299.

[3] Wolpaw, J.R., Vaughan, T.M. and Donchin, E. (1996) EEG based communication prospects and problems. IEEE Transactions on Rehabilitation Engineering, 4, 425-430.

[4] Lotte, F., Congedo, M., Lecuyer, A., Lamarche, F. and Arnaldi, B. (2007) A review of classification algorithms for EEG bases brain computer interface. Journal of neural Engineering, 4, 1-13.

[5] Wolpaw, J.R., Leob, G.E., Allison, B.Z., Donchin, E. and Turner, J.N. (2006) BCI Meeting 2005-Wokshop on signals and rerecording methods. IEEE Transactions on Neural Systems and Rehabilitation Engineering, 14, 138141.

[6] Anderson, C.W., Stolz, E.A. and Shamsunder, S. (1998) Multivariable autoregressive model for classification of spontaneous electroencephalogram during mental tasks. IEEE Transactions on Biomedical Engineering, 45, 277278.

[7] Boostani, R., Graimann, B., Moradi, M.H. and Plurfscheller, G. (2007) Comparison approach toward finding the best feature and classifier in cue BCI. Journal of Medical and Biological Engineering and Computing, 45, 403-413.

[8] Palaniappan, R. (2006) Utilizing gamma band to improve mental task based brain-Computer interface design. IEEE Transactions on Neural and Rehabilitation Systems Engineering, 14, 299-303.

[9] Keirn, Z.A. and Aunon, J.I. (1990) A new mode of communication between man and his Surroundings. IEEE Transactions on Biomedical Engineering, 37, 1209-1214.

[10] Palaniappan, R. (2005) Brain computer interface design using band powers extracted during mental task. Proceeding of the 2nd International IEEE EMBS Conference on Neural Engineering, Arlington, 321-324.

[11] Pfurtscheller, G., Neuper, C., Schlogl, A. and Lugger, K. (1998) Separability of EEG signals recorded during right and left motor imagery using adaptive auto regressive parameters. IEEE Transactions on rehabilitation Engineering, 6, 316-325.

[12] Santhosh, J., Bhatia, M., Sahu, S. and Anand, S. (2004) Quantitative EEG analysis for assessment to plan a task in ALS patients, a study of executive function (planning) in ALS. Journal of Cognitive Brain Research, 22, 59-66.

[13] Nikolaev, A.R. and Anokhin, A.P. (1998) EEG frequency ranges during reception and mental rotation of two and three dimensional objects. Neuroscience and Bheaviour physiology, 29, 203-223.

[14] Osaka, M. (1984) Peak alpha frequency of EEG during a mental task: Task difficulty and hemisphere difference. Journal of Psychophysiology, 21, 101-105.

[15] Ting, W., Zhenga, Y.G., Bang-huaa, Y. and Hong, S. (2008) EEG feature extraction based on wavelet packet 
decom-position for brain computer interface. Measurement, Elsevier Journal, 41, 618-625.

[16] Akay, M. (1995) Wavelet in biomedical engineering. Journal of Annals of Biomedical Engineering, 23, 529530.

[17] Ravi, K.V.R. and Palaniappan, R. (2006) Neural Network
Classification of Late gamma band electroenceph-alogram features, Soft Computing, 10, 163-169.

[18] Cheng, M., Gao, X., Gao, S. and Xu, D. (2002) Design and implementation of a brain computer interface with high transfer rates. IEEE Transactions on Biomedical Engineering, 49, 1181-1186. 